\title{
ÁCIDO ÚRICO Y FACTORES DE RIESGO ASOCIADOS AL SÍNDROME METABÓLICO EN UNA POBLACIÓN ADULTA DE LA CIUDAD DE JUNÍN
}

\author{
Uric acid and risk factors associated with metabolic syndrome in adult population from Junin city \\ Amelia E Carranza ${ }^{1}$, Elena R Benavides ${ }^{2}$, Carmen G Peña ${ }^{2}$, Miguel R Huarcaya ${ }^{2}$, Juan C Quispe ${ }^{1}$, Alejandro A Florentini ${ }^{3}$ \\ ${ }^{1}$ Instituto Nacional de Biología Andina, Facultad de Medicina, UNMSM, 2Facultad de Farmacia y Bioquímica, UNMSM, \\ ${ }^{3}$ Facultad de Ciencias Biológicas, UNMSM
}

\section{RESUMEN}

El objetivo del presente estudio fue determinar la asociación entre niveles séricos de ácido úrico (AU) y factores de riesgo asociados al síndrome metabólico (SM) en una población que habita en grandes alturas. Se incluyeron 174 personas adultas (75 hombres y 99 mujeres), nativas de la ciudad de Junín, $4107 \mathrm{msnm}$. Se determinaron la prevalencia de los siguientes factores de riesgo: obesidad, hipertensión arterial, hiperglucemia, hipertrigliceridemia y valores disminuidos de HDL-colesterol, así como, los niveles séricos de ácido úrico. Los sujetos fueron estratificados, según género, en cuartiles de niveles séricos de ácido úrico. Se encontró una mayor prevalencia de hiperuricemia en hombres que en mujeres. Se observa que la prevalencia de SM aumenta tanto en hombres como en mujeres según los cuartiles de AU sérico. También se encontró que existe asociación significativa entre niveles de ácido úrico y triglicéridos, tanto en hombres como en mujeres. Se concluye que los valores encontrados demostrarían que la prevalencia de síndrome metabólico en los habitantes de grandes alturas está relacionada con el nivel de ácido úrico sérico, por lo que podría ser un nuevo factor de riesgo asociado al SM en estas poblaciones.

Palabras clave: Factor de riesgo, altitud, síndrome metabólico, ácido úrico.

\section{SUMMARY}

The objective of this study was to determine the association between serum uric acid (UA) and risk factors associated with metabolic syndrome (MS) in a high altitude population. We included 174 adults, natives from Junin city, $4107 \mathrm{msnm}$ (75 men and 99 women). The prevalence of risk factors like as obesity, hypertension, hyperglycemia, hypertriglyceridemia and low serum level of HDL-cholesterol and uric acid serum level, were estimated. Subjects were stratified by gender into quartiles of serum uric acid levels. A higher prevalence of hyperuricemia in men than in women was found. It is observed that the prevalence of metabolic syndrome (MS) increases in both men and women according to quartile of serum uric acid (UA). A significant association between uric acid level and triglyceride level in both men and women was found. It is concluded that the estimated values would show the prevalence of MS in people at high altitude to be associated with serum UA level, thus seeming to be a new risk factor associated with the SM in these populations.

Keywords: Risk factor, high altitude, metabolic syndrome, uric acid.

\section{INTRODUCCIÓN}

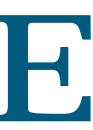
l Síndrome Metabólico (SM) es definido como una serie de factores de riesgo metabólicos que se presentan en conjunto en determinadas personas y que incluyen: resistencia insulínica, obesidad abdominal, hiperglicemia, hipertensión arterial y dislipidemia. Actualmente se le han agregado otros factores como: aumento de LDL pequeñas y densas, ovario poliquístico, hígado graso y aumento del factor inhibidor del activador del plasminógeno (PAI-1).

La prevalencia del SM en una población es variable en función de los criterios de diagnóstico empleados, del grupo étnico estudiado, del sexo y de la distribución por edad.

La National Cholesterol Education Program (NCEP) Adult Treatment Panel III (ATP III) define al SM como la existencia de cualquier combinación de tres alteraciones de los siguientes factores: distribución de grasa corporal, presión arterial, triglicéridos, HDLcolesterol y glicemia en ayunas ${ }^{(1)}$.

El SM se relaciona con un aumento significativo de riesgo de diabetes, enfermedad coronaria y cerebrovascular, transformándose en un factor de riesgo importante de morbimortalidad en la vida adulta ${ }^{(2)}$.

Elácidoúrico (AU) es el producto del metabolismo de las purinas, y sus niveles séricos dependen de una 
serie de factores tales como: dietas con alto contenido en purinas, consumo de alcohol, defectos enzimáticos en el metabolismo de las purinas o una disminución en su excreción.

Cabe indicar que diversos estudios epidemiológicos han señalado la concentración sérica del AU como un importante marcadorderiesgoeneldesarrollo de la enfermedad coronaria cardiaca, cerebrovascular, infarto de miocardio, insuficiencia cardiaca e hipertensión. Además, se ha propuesto que los sujetos con algún desorden del metabolismo purínico pueden ser considerados propensos a una aterogénesis acelerada.

Aunque el $\mathrm{AU}$ no forma parte de la definición de SM, numerosos estudios presentan fuerte asociación entre concentraciones plasmáticas de AU y SM y/o sus componentes (3). Es de considerar que niveles elevados de AU sérico se han encontrado, a menudo, en pacientes con SM, y estudios transversales hechos en varios grupos étnicos han mostrado que la prevalencia del SM se incrementa con el incremento de los niveles de AU sérico ${ }^{(4)}$.

Las poblaciones que viven en "grandes alturas" (GA), es decir por encima de los tres mil metros sobre el nivel del mar, están sometidas a características ambientales especiales. Ello obliga a que en sus organismos se produzcan una serie de cambios adaptativos ${ }^{(5)}$. Se ha demostrado que el poblador de grandes alturas tiene niveles de glicemia más bajos que el poblador del nivel del mar, que la prevalencia de diabetes mellitus es significativamente menor ${ }^{(6)}$, que sus niveles plasmáticos de AU son más altos que que en la gente del nivel del mar (NM) y que tienen una mayor incidencia de gota ${ }^{(7,8)}$.

Estudios fisiológicos, clínicos y epidemiológicos demuestran que hay diferencias, en lo que respecta a
Tabla 1. Características de la población del estudio según cuartil del ácido úrico sérico

\begin{tabular}{|c|c|c|c|c|c|}
\hline & \multicolumn{5}{|c|}{ Hombres } \\
\hline & \multicolumn{5}{|c|}{ Cuartil de ácido úrico sérico } \\
\hline & Q1 & Q2 & Q3 & Q4 & $\mathrm{p}$ \\
\hline Rango de $\mathrm{AU}, \mathrm{mg} / \mathrm{dL}$ & $<5,06$ & $5,15-5,99$ & $6,05-6,94$ & $>7,12$ & \\
\hline $\mathrm{n}$ & 18 & 19 & 19 & 19 & \\
\hline Edad, años & $55,40 \pm 14,3$ & $49,5 \pm 12,1$ & $49,3 \pm 16,6$ & $54,8 \pm 11,0$ & 0,356 \\
\hline Peso, kg & $61,80 \pm 10,1$ & $66,2 \pm 8,3$ & $65,8 \pm 9,9$ & $64,7 \pm 9,7$ & 0,348 \\
\hline Talla, m & $1,65 \pm 0,08$ & $1,64 \pm 0,08$ & $1,66 \pm 0,07$ & $1,60 \pm 0,084$ & 0,166 \\
\hline $\mathrm{IMC}, \mathrm{kg} / \mathrm{m}^{2}$ & $22,66 \pm 2,30$ & $24,88 \pm 2,75$ & $23,85 \pm 2,41$ & $25,19 \pm 3,38$ & $0,026^{*}$ \\
\hline Cintura, cm & $90,40 \pm 6,35$ & $93,6 \pm 8,62$ & $92,4 \pm 6,26$ & $94,3 \pm 6,76$ & 0,373 \\
\hline Cadera, cm & $96,30 \pm 6,13$ & $99,1 \pm 5,02$ & $99,3 \pm 6,11$ & $99,6 \pm 5,14$ & 0,273 \\
\hline P. sistólica, mmHg & $119,30 \pm 15,7$ & $123,5 \pm 13,0$ & $120,4 \pm 14,4$ & $132,2 \pm 22,8$ & 0,091 \\
\hline P. diastólica, mmHg & $75,70 \pm 11,3$ & $79,2 \pm 8,4$ & $79,7 \pm 10,7$ & $88,3 \pm 18,5$ & $0,026^{*}$ \\
\hline Glucosa, mg/dL & $80,20 \pm 22,2$ & $79,3 \pm 15,8$ & $74,4 \pm 16,5$ & $85,4 \pm 40,0$ & 0.628 \\
\hline Triglicéridos, mg/dL & $107,20 \pm 65,2$ & $201,7 \pm 119,3$ & $157,9 \pm 103,8$ & $217,0 \pm 94,8$ & $0,006^{*}$ \\
\hline Colesterol, mg/dL & $140,2 \pm 24,4$ & $152,5 \pm 29,9$ & $158,0 \pm 31,2$ & $183,1 \pm 20,6$ & $0,000^{*}$ \\
\hline HDL-col, mg/dL & $41,0 \pm 11,4$ & $36,2 \pm 7,8$ & $35,3 \pm 10,4$ & $36,1 \pm 11,4$ & 0,339 \\
\hline LDL-col, mg/dL & $77,7 \pm 24,0$ & $76,0 \pm 34,4$ & $91,0 \pm 39,2$ & $105,1 \pm 25,1$ & $0,027^{*}$ \\
\hline \multirow[t]{4}{*}{ Ácido úrico, mg/dL } & $4,33 \pm 0,55$ & $5,55 \pm 0,27$ & $6,45 \pm 0,28$ & $8,45 \pm 1,04$ & 0 \\
\hline & \multicolumn{5}{|c|}{ Mujeres } \\
\hline & \multicolumn{5}{|c|}{ Cuartil de ácido úrico sérico } \\
\hline & Q1 & Q2 & Q3 & Q4 & p \\
\hline Rango de $\mathrm{AU}, \mathrm{mg} / \mathrm{dL}$ & $<3,78$ & $3,81-4,54$ & $4,55-5,39$ & $>5,45$ & \\
\hline $\mathrm{n}$ & 24 & 25 & 25 & 25 & \\
\hline Edad, años & $45,0 \pm 13,2$ & $50,2 \pm 10,4$ & $43,8 \pm 10,6$ & $50,6 \pm 13,4$ & 0,103 \\
\hline Peso, kg & $52,6 \pm 7,5$ & $54,7 \pm 6,2$ & $59,0 \pm 14,7$ & $60,2 \pm 11,2$ & $0,040^{*}$ \\
\hline Talla, m & $1,497 \pm 0,05$ & $1,509 \pm 0,05$ & $1,514 \pm 0,09$ & $1,504 \pm 0,08$ & 0.835 \\
\hline $\mathrm{IMC}, \mathrm{kg} / \mathrm{m}^{2}$ & $23,48 \pm 3,39$ & $24,06 \pm 3,06$ & $25,62 \pm 5,31$ & $26,65 \pm 4,68$ & $0,040^{*}$ \\
\hline Cintura, cm & $89,0 \pm 9,3$ & $91,0 \pm 8,6$ & $93,9 \pm 11,2$ & $94,0 \pm 9,8$ & 0,223 \\
\hline Cadera, cm & $98,6 \pm 7,3$ & $101,0 \pm 7,3$ & $103,5 \pm 10,1$ & $104,4 \pm 9,9$ & 0,101 \\
\hline P. sistólica, mmHg & $110,1 \pm 17,9$ & $111,2 \pm 18,1$ & $111,4 \pm 20,2$ & $122,6 \pm 18,1$ & 0,065 \\
\hline P. diastólica, mmHg & $78,8 \pm 9,1$ & $76,3 \pm 13,2$ & $81,6 \pm 14,7$ & $83,8 \pm 13,1$ & 0,185 \\
\hline Glucosa, mg/dL & $80,0 \pm 11,5$ & $77,3 \pm 11,0$ & $87,8 \pm 40,9$ & $83,3 \pm 10,9$ & 0,411 \\
\hline Triglicéridos, mg/dL & $117,4 \pm 48,1$ & $133,4 \pm 82,1$ & $148,6 \pm 86,5$ & $221,3 \pm 127,8$ & $0,001^{*}$ \\
\hline Colesterol, mg/dL & $140,4 \pm 25,0$ & $147,0 \pm 29,8$ & $158,8 \pm 34,2$ & $172,4 \pm 39,8$ & $0,005^{*}$ \\
\hline HDL-col, mg/dL & $41,3 \pm 11,4$ & $42,7 \pm 9,7$ & $44,9 \pm 14,1$ & $41,2 \pm 9,2$ & 0,628 \\
\hline LDL-col, mg/dL & $75,6 \pm 23,3$ & $77,6 \pm 30,2$ & $84,3 \pm 32,6$ & $87,0 \pm 36,7$ & 0,531 \\
\hline Ácido úrico, mg/dL & $3,34 \pm 0,37$ & $4,23 \pm 0,25$ & $4,88 \pm 0,27$ & $6,36 \pm 0,93$ & 0 \\
\hline
\end{tabular}
ivo

enfermedad coronaria, entre los residentes NM y los de GA, teniendo estos últimos una baja incidencia ampliamente conocida. De los factores de riesgo implicados se conoce que en los habitantes de GA, la tasa de colesterol es menor y los niveles de HDLcolesterol mayor que a nivel del mar.

También se ha observado una menor frecuencia de sobrepeso y menor prevalencia de hipertensión en las poblaciones nativas de altura ${ }^{(6)}$.

Sobre una posible asociación entre el nivel sérico de $\mathrm{AU}$ y los diversos componentes del SM en sujetos de grandes alturas, no hemos encontrado ninguna referencia en la bibliografía revisada, por lo que el 
Tabla 2. Prevalencia de cada factor asociada al SM según el cuartil de ácido úrico sérico.

\begin{tabular}{|c|c|c|c|c|c|}
\hline & \multicolumn{5}{|c|}{ Hombres } \\
\hline & \multicolumn{5}{|c|}{ Cuartil de ácido úrico sérico } \\
\hline & Q1 & Q2 & Q3 & Q4 & $\mathrm{p}$ \\
\hline Rango de $\mathrm{AU}, \mathrm{mg} / \mathrm{dL}$ & $<5,06$ & $5,15-5,99$ & $6,05-6,94$ & $>7,12$ & \\
\hline $\mathrm{n}$ & 18 & 19 & 19 & 19 & \\
\hline Síndrome metabólico & $0(0 \%)$ & $2(10,5 \%)$ & $3(15,8 \%)$ & $6(31,6 \%)$ & $0,037^{*}$ \\
\hline IMC & $0(0 \%)$ & $2(10,5 \%)$ & $0(0 \%)$ & $2(10,5 \%)$ & 0,249 \\
\hline Obesidad central & $9(50 \%)$ & $13(68,4 \%)$ & $16(84,2 \%)$ & $15(78,9 \%)$ & 0,108 \\
\hline P. sistólica alta & $6(33,3 \%)$ & $6(31,6 \%)$ & $5(26,3 \%)$ & $8(42,1 \%)$ & 0,776 \\
\hline Hiperglicemia & $2(11,1 \%)$ & $3(15,8 \%)$ & $2(10,5 \%)$ & $3(15,8 \%)$ & 0,939 \\
\hline Hipertrigliceridemia & $3(16,7 \%)$ & $11(57,9 \%)$ & $8(44,4 \%)$ & $11(64,7 \%)$ & $0,021^{*}$ \\
\hline \multirow[t]{4}{*}{ HDL-col bajo } & $13(72,2 \%)$ & $18(94,7 \%)$ & $17(94,4 \%)$ & $15(83,3 \%)$ & 0,151 \\
\hline & \multicolumn{5}{|c|}{ Mujeres } \\
\hline & \multicolumn{5}{|c|}{ Cuartil de ácido úrico sérico } \\
\hline & Q1 & Q2 & Q3 & Q4 & $\mathrm{p}$ \\
\hline Rango de $\mathrm{AU}, \mathrm{mg} / \mathrm{dL}$ & $<3,78$ & $3,81-4,54$ & $4,55-5,39$ & $>5,45$ & \\
\hline $\mathrm{n}$ & 24 & 25 & 25 & 25 & \\
\hline Síndrome metabólico & $2(8,3 \%)$ & $3(12,0 \%)$ & $8(32,0 \%)$ & $12(52,0 \%)$ & $0,001^{*}$ \\
\hline IMC & $1(4,2 \%)$ & $1(4,0 \%)$ & $3(12,0 \%)$ & $5(20,0 \%)$ & 0,191 \\
\hline Obesidad central & $2(8,3 \%)$ & $2(8,0 \%)$ & $3(12,0 \%)$ & $6(24,0 \%)$ & 0,298 \\
\hline P. sistólica alta & $3(12,5 \%)$ & $5(20,0 \%)$ & $3(12,0 \%)$ & $9(36,0 \%)$ & 0,122 \\
\hline Hiperglicemia & $1(4,5 \%)$ & $1(4,0 \%)$ & $3(12,0 \%)$ & $1(4,0 \%)$ & 0,579 \\
\hline Hipertrigliceridemia & $5(20,8 \%)$ & $7(28,0 \%)$ & $11(44,0 \%)$ & $17(68,0 \%)$ & $0,004^{*}$ \\
\hline
\end{tabular}

* Significativos: Hombres.- Obesidad central en Q3 respecto de Q1; Hipertrigliceridemia en Q2 y Q4 respecto de Q1 Mujeres.- Hipertrigliceridemia en Q4 respecto de Q1.

Tabla 3. OR (IC $95 \%$ ) entre ácido úrico y componentes del SM.

\begin{tabular}{lcccc}
\hline & \multicolumn{4}{c}{ Cuartiles de ácido úrico sérico } \\
\cline { 2 - 5 } HOMBRES & $\mathbf{Q 1}$ & $\mathbf{Q 3}$ & $\mathbf{Q 4}$ \\
$\quad$ Obesidad & 1 & $0,84(0,84-0,84)$ & $0,84(0,84-0,84)$ & $1,00(0,95-1,05)$ \\
Obesidad central & 1 & $2,40(0,60-9,44)$ & $5,97(1,23-29,01)$ & $3,79(0,90-16,06)$ \\
Presión sistólica alta & 1 & $0,85(0,21-3,45)$ & $0,66(0,16-2,77)$ & $1,44(0,37-5,56)$ \\
Hiperglicemia & 1 & $1,77(0,25-12,66)$ & $3,11(0,13-9,22)$ & $1,54(0,22-10,68)$ \\
Hipertrigliceridemia & 1 & $6,29(1,34-29,69)$ & $3,60(0,75-17,24)$ & $9,45(1,93-46,81)$ \\
HDL-col bajo & 1 & $2,80(0,46-17,34)$ & $5,59(0,56-55,25)$ & $1,56(0,38-10,04)$ \\
MUJERES & & & & \\
Obesidad & & & & \\
Obesidad central & 1 & $0,75(0,04-13,23)$ & $3,42(0,32-36,27)$ & $4,65(0,48-44,58)$ \\
Presión sistólica alta & 1 & $0,91(0,12-7,13)$ & $1,53(0,23-10,14)$ & $3,30(0,58-18,68)$ \\
Hiperglicemia & 1 & $1,46(0,30-7,21)$ & $1,00(0,18-5,66)$ & $3,36(0,76-14,83)$ \\
Hipertrigliceridemia & 1 & $0,69(0,04-12,36)$ & $3,01(0,28-31,83)$ & $0,67(0,037-12,16)$ \\
HDL-col bajo & 1 & $1,15(0,29-4,56)$ & $3,64(0,96-13,83)$ & $7,16(1,86-27,54)$ \\
\hline
\end{tabular}

Significativos: $\quad$ Hombres.- Obesidad central en Q3 respecto de Q1; Hipertrigliceridemia en Q2 y Q4 respecto de Q1 Mujeres.- Hipertrigliceridemia en Q4 respecto de Q1.

Tabla 4. OR (IC $95 \%$ ) entre ácido úrico y SM, ajustado para edad.

\begin{tabular}{lccccc}
\hline & \multicolumn{5}{c}{ Cuartiles de ácido úrico sérico } \\
\cline { 2 - 6 } & Q1 & Q2 & Q3 & Q4 \\
\hline Hombres & 1 & $1,03(0,98-1,08)$ & $1,00(0,13-7,97)$ & $4,00(0,68-23,50)$ \\
Mujeres & 1 & $0,41(0,04-4,92)$ & $5,59(1,03-30,38)$ & $10,81(2,06-56,74)$ \\
& Total & 1 & $1,46(0,23-9,22)$ & $6,11(1,25-29,95)$ & $15,28(3,27-71,45)$ \\
\hline
\end{tabular}

Significativos: Mujeres en Q3 y en Q4 respecto de Q1; y el total en Q3 y en Q4 respecto de Q1. 
propósito del presente estudio fue determinar si el nivel de acido úrico sérico está asociado a marcadores de riesgo de Síndrome Metabólico en este tipo de población.

\section{MATERIAL Y MÉTODOS}

\section{Sujetos de experiencia}

El presente es un estudio transversal en el que se incluyeron a 174 personas adultas, 75 hombres y 99 mujeres, con una edad media de 50,5 y 47,4 años, respectivamente, nativas de la ciudad de Junín, 4107 msnm.

Los sujetos de estudio se presentaron a primera hora de la mañana para la toma de muestra respectiva en condiciones de ayuno absoluto (12 horas previas) y sin haber realizado actividad física.

\section{Recolección de la muestra}

Las muestras sanguíneas, $8 \mathrm{ml}$, fueron obtenidas mediante punción venosa del antebrazo previa asepsia, siendo recibidas en tubos de ensayo sin anticoagulante, limpios y desinfectados. La sangre extraída se dejó reposar por 30 minutos para coagular y luego se centrifugó por 5 minutos a $4000 \mathrm{rpm}$ para separar el suero. Estos fueron colocados en un recipiente hermético entre 2 a $5^{\circ} \mathrm{C}$, transportándose al laboratorio del Instituto Nacional de Biología Andina, para ser almacenados $\mathrm{a}-40^{\circ} \mathrm{C}$, hasta el momento del análisis.

\section{Mediciones}

La toma de la presion arterial la efectuó una profesional de la salud; se pesaron y tallaron a los individuos, sin calzado y con ropa ligera, asimismo, se midieron los perímetros de la cintura y cadera, calculándose finalmente el índice de masa corporal (IMC) según la fórmula Quetelet (peso en $\mathrm{kg}$ dividido entre el cuadrado de la talla en metros).

\section{Determinaciones}

En el suero se determinaron: glucosa, colesterol total, HDL-colesterol, triglicéridos y ácido úrico por métodos enzimáticos convencionales y con kits comerciales. La concentración sérica de LDL-colesterol se determinó aplicando la fórmula de Friedewald.

El diagnóstico del SM fue realizado de acuerdo al criterio del National Cholesterol Education Program (NCEP) Adult Treatment Panel III (ATP III) ${ }^{(1)}$. Se consideró SM a la presencia de tres o más de los siguientes criterios: triglicéridos $\geq 150 \mathrm{mg} / \mathrm{dL}$; HDLcolesterol $<40 \mathrm{mg} / \mathrm{dL}$ en hombres $\mathrm{y}<50 \mathrm{mg} / \mathrm{dL}$ en mujeres; presión arterial $\geq 130 / 85 \mathrm{mmHg}$, glucemia en ayunas $\geq 110 \mathrm{mg} / \mathrm{dL}$ y circunferencia de la cintura $>102 \mathrm{~cm}$ para hombres y $>88 \mathrm{~cm}$ para las mujeres. La Organización Mundial de la Salud considera a la obesidad como uno de los mayores problemas de salud pública del siglo XXI, por lo que se incluye el índice de masa corporal (IMC) como un criterio adicional (IMC $>$ a $\left.30 \mathrm{~kg} / \mathrm{m}^{2}\right)$.

Se define hiperuricemia a una concentración sérica de ácido úrico mayor a 7,0 $\mathrm{mg} / \mathrm{dL}$ en hombres y $5,7 \mathrm{mg} / \mathrm{dL}$ en mujeres.

Los sujetos fueron estratificados según género en cuartiles de niveles séricos de ácido úrico.

\section{Análisis estadístico}

Los resultados son expresados en valores medios y desviación estándar, y frecuencias relativas de casos. Se efectuó la comparación de medias según el análisis de varianza y la comparación de proporciones mediante la prueba chi-cuadrado. El cociente de las probabilidades [Odds Ratio (OR)], con un intervalo de confianza del 95\% (IC 95\%), fue calculado para cada nivel de ácido úrico sérico, tomando el cuartil más bajo como referencia y utilizando modelos de regresión logística multinomial. Se consideró significativo todo resultado cuyo valor asociado de $\mathrm{p}$ fuese $<0,05$. El análisis estadístico se llevó a cabo mediante el programa SPSS versión 15,0.

\section{RESULTADOS}

La media, \pm desviación estándar del nivel de ácido úrico sérico, fue 6,22 $\pm 1,64 \mathrm{mg} / \mathrm{dL}$ en hombres y 4,72 $\pm 1,22 \mathrm{mg} / \mathrm{dL}$ en mujeres. En la población estudiada se encontró una prevalencia de hiperuricemia de $25,33 \%$ en hombres y $16,16 \%$ en mujeres.

Los valores de $\mathrm{AU}$ se agruparon en cuartiles, tomando como base los valores obtenidos en ayunas, (hombres: el primero < 5,06 mg/dL, el segundo 5,15 a $5,99 \mathrm{mg} / \mathrm{dL}$, el tercero 6,05 a 6,94 mg/dL y el cuarto cuartil > 7,12 mg/dL, y mujeres: el primero $<3,78 \mathrm{mg} /$ dL, el segundo 3,81 a 4,54 mg/dL, el tercero 4,55 a 5,39 $\mathrm{mg} / \mathrm{dL}$ y el cuarto cuartil $>5,45 \mathrm{mg} / \mathrm{dL}$ ).

En la tabla 1 se presentan los resultados del análisis de varianza de los parámetros estudiados según cuartiles de AU. Podemos observar que los incrementos de AU se asocian con el IMC, niveles de triglicéridos y colesterol total, tanto en hombres como en mujeres. En las mujeres los cuartiles de AU se asocian al peso, 


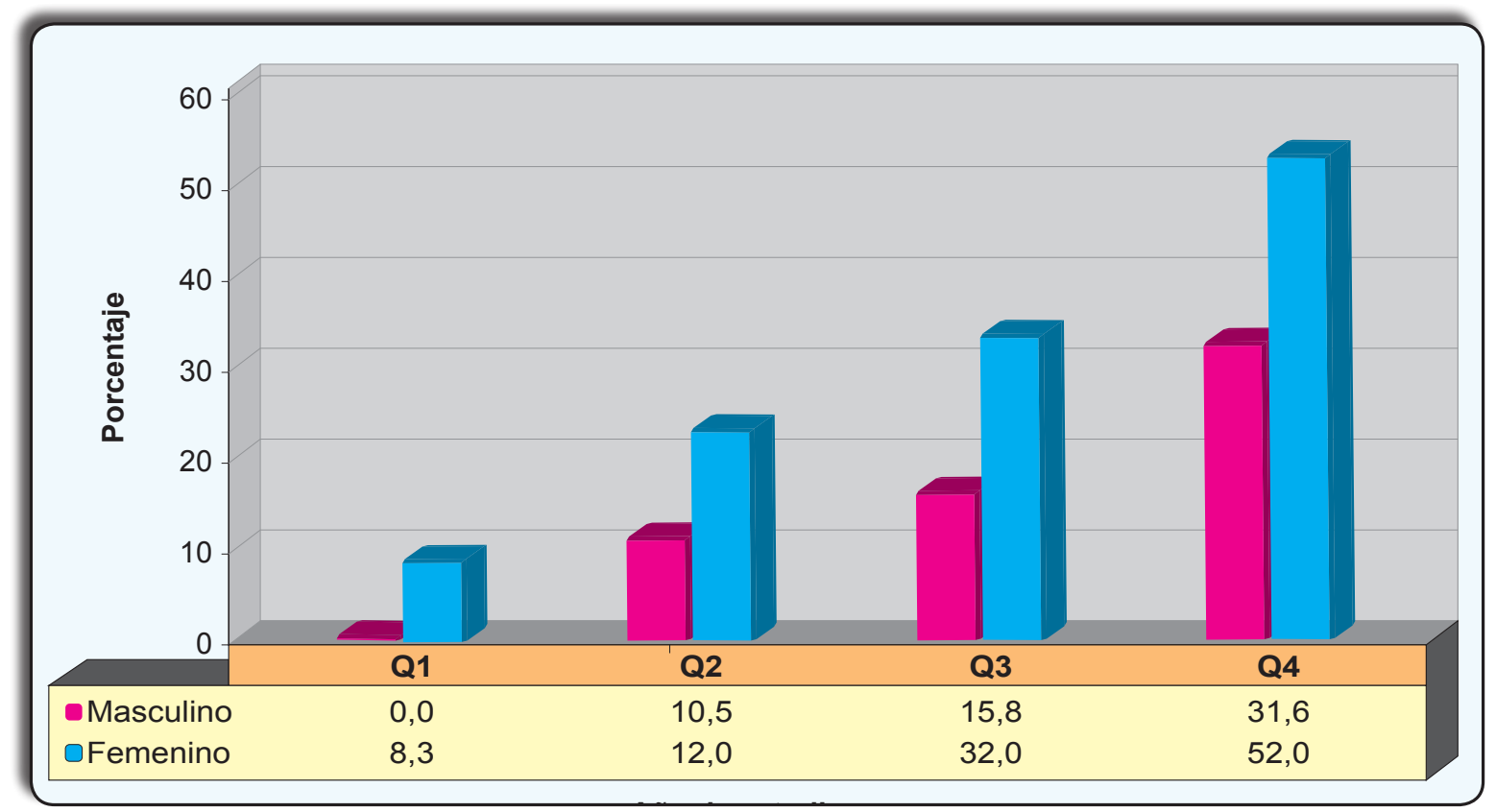

Figura 1. Prevalencia de SM de acuerdo al cuartil de acido úrico sérico.

mientras que en los hombres se observa una relación entre cuartiles de $\mathrm{AU}$, presión diastólica y niveles de LDL-colesterol.

En la Tabla 2 se presentan las prevalencias de SM y de cada componente asociado al SM según cuartil de AU sérico. Se observa que la prevalencia de SM aumenta tanto en hombres como en mujeres según el cuartil de AU sérico ( $p=0,037$ y 0,001 , respectivamente) (Fig. 1). La prevalencia de SM en el cuarto cuartil fue mayor que en el primero en mujeres y segundo de hombres; no se encontró ningún caso de SM en el primer cuartil de hombres; el incremento de la prevalencia en mujeres es mayor que en hombres en todos los cuartiles.

Por otro lado, se investigó cuál de los componentes del SM es más prevalente, y encontramos que el nivel disminuido de HDL-colesterol es mayor en el caso del género masculino en todos los cuartiles, pero sin significación estadística, mientras que en el femenino, el mayor indicador de riesgo es el nivel de triglicéridos séricos basales en el cuarto cuartil $(p=0,004)$.

También seevaluóel cocientedelas probabilidades (Odds Ratio [OR]), con un intervalo de confianza del $95 \%$ (IC 95\%), de los niveles de acido úrico y para cada componente del SM tomando el cuartil más bajo como referencia, utilizando modelos de regresión logística multivariable. Se observó una asociación positiva entre los niveles séricos de AU y obesidad central en hombres, pero no en mujeres, y AU y triglicéridos tanto para hombres como para mujeres (Tabla 3).

En relación al SM (Tabla 4), los resultados demuestran que las mujeres del tercery cuarto cuartiles presentan mayor riesgo de síndrome metabólico que las del primer cuartil: 59 y 10,81 veces, respectivamente. Estos valores son significativos cuando el intervalo de confianza no incluye el valor 1. En hombres, si bien el cuarto cuartil presenta un valor incrementado de riesgo, éste no presenta diferencia significativa en relación al primero.

\section{DISCUSIÓN}

En el presente trabajo hemos efectuado el análisis de la relación de seis factores de riesgo, componentes del síndrome metabólico, cinco de acuerdo con los criterios del ATPIII, y uno (IMC) de acuerdo a la OMS, en hombres y mujeres oriundos de la ciudad de Junín $(4107 \mathrm{~m})$, con los valores séricos de acido úrico basal.

Los niveles medios de ácido úrico sérico de la población masculina y femenina fueron 6,22 y 4,72 mg/ $\mathrm{dL}$, respectivamente. La prevalencia de hiperuricemia en hombres fue de $25,3 \%$ y en mujeres $9,7 \%$, menores de 45 años y 19,0\% en mayores de esa edad. Estos resultados concuerdan con diversos autores que encuentraron que la menor concentración de AU en mujeres premenopáusicas se debe a la influencia hormonal, ya que se conoce que los estrógenos promueven el aclaramiento renal más eficiente del $\mathrm{AU}{ }^{\left({ }^{9}\right.}$. Estudios epidemiológicos han sugerido que el 
aumento de ácido úrico sérico relacionado con la edad en mujeres se debe a la menopausia. Esta hipótesis ha sido confirmada por la reducción de urato sérico después de usar la terapia de reemplazo hormonal en mujeres posmenopáusicas ${ }^{(10,11)}$.

Niveles séricos de AU elevados se observan con frecuencia en asociación con factores individuales de riesgo cardiovascular tales como la obesidad, hiperglucemia, hipertensión e hipertrigliceridemia. En nuestro estudio, de los seis componentes analizados, solamente la hipertrigliceridemia presenta asociación con el incremento de AU; este hallazgo está de acuerdo con otros estudios, que han encontrado que el nivel de TG se correlaciona de forma independiente con el nivel de $\mathrm{AU}^{(12,13)}$.

El mecanismo de esta asociación no ha sido dilucidado completamente, se sugiere que hiperuricemia y valores altos de TG se relacionan con el síndrome de resistencia a la insulina debido a que la glucólisis es controlada por la insulina y la producción de ácido úrico está vinculada a la glucólisis ${ }^{(14)}$. La síntesis de los nucleótidos de purina comienza con el 5-fosforibosil-1-pirofosfato (PPRP) que se forma a partir de la ribosa $5-\mathrm{P}(\mathrm{R}-5-\mathrm{P})$ a través de la vía de las pentosas y cuya producción se rige por el flujo glucolítico. Se ha encontrado que una disminución de la actividad enzimática de la gliceraldehído-3-fosfato deshidrogenasa $\left(\mathrm{GA}_{3} \mathrm{PDH}\right)$, que es regulada por la insulina, produce una acumulación de metabolitos intermedios y una desviación de éstos hacia la síntesis de R-5-P, PPRP, y ácido úrico. La disminución de la actividad de dicha enzima favorece también la acumulación de glicerol-3-fosfato, lo que permitiría incrementar la concentración de triglicéridos séricos. Por lo tanto, los defectos intrínsecos en $\mathrm{GA}_{3} \mathrm{PDH}$ o la pérdida de su capacidad de respuesta a la insulina, permitiendo que se acumulen los intermediarios glucolíticos, puede explicar la asociación entre la resistencia a la insulina, hiperuricemia, e hipertrigliceridemia ${ }^{(15)}$.

Por otro lado, aunque factores genéticos están asociados con la presencia degota ehipertrigliceridemia (2), muchos investigadores han concluido que la hiperuricemia y la hipertrigliceridemia reflejan el estilo de vida del paciente más que los factores genéticos (16), porque la obesidad también se asocia con estas características y, en nuestro caso, la hiperuricemia se asoció con el peso en mujeres, aunque no en hombres.

La falta de asociación entre AU y glucosa, y presión arterial, se debería a que en la altura normalmente los valores medios de glucosa sérica son menores que sus similares del nivel mar, y también se ha reportado una menor prevalencia de hipertensión. En este estudio se encontraron siete casos de hiperglucemia en hombres y un solo caso en mujeres.

En el presente estudio se encontró un incremento de la prevalencia del síndrome metabólico tanto en hombres como en mujeres relacionado con el aumento de los niveles séricos de AU. Las mujeres tuvieron mayor incremento de prevalencia en el cuarto cuartil de AU (52\%) en relación con los hombres (32\%).

La prevalencia en el primero y segundo cuartiles son o y $2 \%$ en hombres y, 2 y $3 \%$ en mujeres, respectivamente, lo que demuestra que la prevalencia del síndrome metabólico está relacionado con los niveles de acido úrico sérico.

También se evaluó la odds ratio (OR) y el intervalo de confianza del 95\% (IC 95\%) de los niveles séricos de ácido úrico y SM (ajustado para edad) con cada uno de sus componentes. Se encontró que el AU estaba asociado principalmente con la hipertrigliceridemia, tanto en hombres como en mujeres. En el caso de los hombres los OR (IC 95\%) en el segundo y cuarto cuartiles fueron: 6,29 (1,33-29,69) y 9,45 (1,90$46,89)$, es decir, que los hombres con niveles de AU sérico en el segundo y cuarto cuartiles presentaron, respectivamente, 6,29 y 9,45 veces mayor riesgo que en el primer cuartil, y en el caso de las mujeres del cuarto cuartil el OR (IC 95\%) fue: 7,16 (1,86-27,54), es decir 7,16 veces mayor riesgo con respecto al primer cuartil.

$\mathrm{Al}$ ajustar el OR para la edad se encontró que en los hombres el cuarto cuartil de AU presenta un valor incrementado de riesgo, pero no se observa asociación significativa entre AU sérico y SM; en el caso de las mujeres se halló que con niveles de $\mathrm{AU}$ de $>4,55^{-}$ $5,39 \mathrm{mg} / \mathrm{dL}$ en el tercer cuartil y $>5,45 \mathrm{mg} / \mathrm{dL}$ en el cuarto, tuvieron un aumento significativo en el riesgo de síndrome metabólico (OR: 5,59 y 10,81), lo cual confirma que la asociación de ácido úrico y SM es mas estrecha en mujeres que en hombres.

En conclusión, hay una significativa asociación entre niveles de acido úrico sérico y síndrome metabólico en pobladores de la ciudad de Junín, principalmente en mujeres, quienes con niveles de AU en el cuarto cuartil $>5,45 \mathrm{mg} / \mathrm{dL}$ tienen un alto riesgo de desarrollar síndrome metabólico. Se indica además que los niveles séricos de triglicéridos están fuertemente asociados a los niveles de acido úrico, tanto en hombres como en mujeres. 


\section{REFERENCIAS BIBLIOGRÁFICAS}

1. Grundy SM, Brewer H, Cleeman J, Smith S, Lenfant C. Definition of metabolic syndrome: Report of the National Heart, Lung, and Blood Institute/American Heart Association conference on scientific issues related to definition. Circulation 2004;109:433-8.

2. Alexander CM, Landsman P, Teutsch M, Haffner S. NCEPDefined metabolic syndrome, diabetes and prevalence of coronary heart disease among NHANES III participants age 50 and older. Diabetes 2003; 52: 1210-4.

3. Lin SH, Tsai DH, Hsu SR. Association between serum uric acid level and components of the metabolic syndrome. J Chin Med Assoc 2006; 69: 512-6.

4. LimJH, Kim YK, Kim YS, NaSN, RheeMY, LeeMM. Relationship between serum uric acid levels, metabolic syndrome, and arterial stiffness in korean. Korean Circ J 2010; 40: 314-20.

5. West JB, Schoene RB, Milledge JS. High altitude medicine and physiology. $4^{\mathrm{a}}$ ed. Hodder Arnold a Member of the Hodder Headline Group. London, 2007.

6. Gonzáles G. Metabolismo en las grandes alturas. Acta Andina 2001; 9: 31-42.

7. Hurtado AA, Fuquay R, Sirota J, Swenson E, Schoene R, Jefferson J, et al. High Altitude Renal Syndrome (HARS). J Am Soc Nephrol 2011; 22: 1963-8.

8. Jefferson JA, Escudero E, Hurtado ME, Kelly JP, Swenson ER, Wener MH, et al. Hyperuricemia, hypertension, and proteinuria associated with high-altitude polycythemia. Am J Kidney Dis 2002; 39: 1135-42.

9. Nicholls A, Snaith M, Scott J. Effect of Oestrogen Therapy on Plasma and Urinary Levels of Uric Acid. Br Med J 1973;1: 449-51.

10. Sumino H, Ichikawa S, Kanda T, Nakamura T, Sakamaki $\mathrm{T}$. Reduction of serum uric acid by hormone replacement therapy in postmenopausal women with hyperuricaemia. Lancet 1999; 354: 650.

11. Hak EA, Choi HK. Menopause, postmenopausal hormone use and serum uric acid levels in US women - The third national health and nutrition examination survey. Arthritis Res. Ther. 2008; 10:R116.

12. Saleh Saneya A, Al-Muammar M. Relation between Hyperuricemia and Metabolic Syndrome among Obese Women. JMRI 2009; 30: 91-7

13. Hayden MR, Tyagi SC. Uric acid: A new look at an old risk marker for cardiovascular disease, metabolic syndrome, and type 2 diabetes mellitus: The urate redox shuttle. Nutr Metab. 2004; 1: 10.

14. RathmannW, FunkhouserE, DyerAR, RosemanJM. Relations of hyperuricemia with the various components of the insulin resistance syndrome in young black and white adults: the CARDIA study. Coronary Artery Risk Development in Young Adults. Ann Epidemiol 1998; 8:250-61.

15. Leyva F, Wingrove S, Godsland I, Stevenson, J. The glycolytic pathway to coronary heart disease: a hypothesis. Metabolism 1998; 47: 657-62.

16. Roddy E. Hyperuricemia, gout, and lifestyle factors. J Rheumatol. 2008; 35:1689-91.

Manuscrito recibido el: 02/11/11

Aceptado para su publicación el: 04/01/2012

\section{Correspondencia:}

Nombre: $\quad$ Elizabeth Carranza Alva

Dirección: Jr. Puno 1002- Lima 1 - Perú

e-mail: ecarranzalva@yahoo.com 